

\title{
Introduction and Propagation Properties \\ of Circular Lorentz-Bessel-Gaussian beams
}

\author{
Ahmed Abdulrab Ali Ebrahim ${ }^{1,2}$, Nabil A.A. Yahya ${ }^{2,3}$, \\ Mohamad Swillam², Abdelmajid Belafhal ${ }^{4, *}$ \\ ${ }^{1}$ Ministry of Education, Taiz, Yemen \\ ${ }^{2}$ Department of Physics, School of Sciences and Engineering, \\ The American University in Cairo, New Cairo, Cairo, 11835, Egypt \\ ${ }^{3}$ Thamar University, Department of Physics, Thamar, Yemen \\ ${ }^{4}$ Laboratory LPNAMME, Laser Physics Group, \\ Department of Physics, Faculty of Sciences, Chouaïb Doukkali University, \\ P. B 20, 24000 El Jadida, Morocco \\ *Corresponding author. E-mail: belafhal@gmail.com
}

\begin{abstract}
In this paper, circular Lorentz-Bessel-Gaussian beams (CLBGBs) are introduced as a novel member of the Lorentz-Gaussian beams family. The analytical expression of the propagation of these beams passing through a paraxial optical ABCD system is derived. The generalized Huygens-Fresnel diffraction integral of the form Collins's formula and the expansion of the Lorentz distribution in terms of the complete orthonormal basis set of the Hermite-Gauss modes are used. The influences of the beam-order and Bessel part $\beta$, Gaussian and Lorentzian waists, and propagation distance $\mathrm{z}$ on the propagation of CLBGBs are investigated. Some numerical simulation results are done. The beams family in this work may be useful to the practical applications in free-space optical communications because they have vortex properties with their being experimentally realizable.
\end{abstract}

Keywords: Circular Lorentz-Bessel-Gaussian beams; Paraxial ABCD optical system; Collins's formula.

\section{Introduction}

As new realizable beams, Gawhary and Severini introduced Lorentz and Lorentz-Gauss beams (Gawhary and Severini 2006; 2007). The angular spread of these beams was higher than that of the pure Gaussian beams (Dumke 1975). Therefore, the beams family of Lorentz and Lorentz-Gauss beams provide more appropriate models than Gaussian to describe certain laser 
sources (Naqwi and Durst 1990, Yang et al. 2008). They were valuable to characterize diode laser wavelength (Xu, and Zhou 2019). Consequently, much literature has been published about the propagation properties of this beams family. Based on the Fresnel approximation, the propagation properties of Lorentz-Gauss beams in free space have been investigated (Gawhary and Severini 2006). The propagation of a partially coherent Lorentz-Gauss beam through the aligned/misaligned ABCD optical system has been studied in detail, respectively (Zhou 2010a, 2010b). The properties of Lorentz or Lorentz-Gauss beams after propagating through multi-slits of Fractional Fourier transform planes have been also reported by other authors (Zhou 2009a; Du et al. 2011). The Irradiance fluctuations of partially coherent super Lorentz Gaussian beams were examined (Muhsin et al. 2011). The paraxial propagation of Lorentz and Lorentz-Gauss beams in uniaxial crystals orthogonal to the optical axis has been developed (Zhao and Cai 2010).

Moreover, the beam quality, the kurtosis characteristics and the linearly polarized LorentzGauss beam focused with one optical vortex have been studied (Zhou 2009b, c, Rui et al. 2013). However, Lorentz and Lorentz-Gaussian beams have poor symmetry. To overcome this defect, Xu and Zhou, 2019 have proposed a circular Lorentz-Gauss beam (CLGB) beam and they have studied the propagation properties of the beam through a paraxial ABCD optical system and its parametric characterization.

Bessel-Gaussian beams have received a lot of attention because they show interesting optical properties on the diffraction path such as an extended depth of field, an annular intensity distribution (Schimpf et al. 2012). Therefore, a circular Lorentez-Bessel-beam modulated by a fundamental Gaussian beam will become more beneficial in optical techniques (Fahrbach et al. 2010; Planchon and Gao 2011; Duocastella and Arnold 2012; Garcés-Chávez et al. 2002; Li and Imasaki 2005).

In the present work, a novel family of Lorentz optical beams so-called Circular LorentzBessel-Gaussian beams was introduced. We applied the Collins's formula to find an output field given in terms of the Hermite-Gauss modes. But the problem of this optical translation was mainly confined to the derivation of the output analytical expression result. The early integral result provided by Belafhal et al., 2020 in mathematics was used to solve that problem. Also, the expansion of the Lorentz distribution in terms of the complete orthonormal basis set of the Hermite-Gauss modes (Schmidt 1976) was used. The effect of introducing the general Bessel beam of the first kind into the Circular Lorentz Gaussian beam will be examined. The closed-form 
expression of their propagation through an ABCD optical system using the Collins's formula (1970) will be derived to obtain the receiver field.

\section{Expression of CLBGBs}

In this Section, we use Eq. (5) of Ref. (Schmidt 1976) to deduce the amplitude field of CLGB, which is the incident field in our analytical treatment. From the above reference, the expansion of the Lorentz distribution can be written as

$$
L=\frac{\gamma}{2 \pi\left(y^{2}+\gamma^{2} / 4\right)}=\sqrt{2 / \pi} \frac{1}{\alpha \gamma} \sum_{n=0}^{N} a_{2 n}(\alpha) H_{2 n}\left(\frac{2 y}{\alpha \gamma}\right) e^{-\frac{2 y^{2}}{\alpha^{2} \gamma^{2}}},
$$

where $H_{2 n}(\cdot)$ is the Hermite polynomial of even order, $N$ is the number of the expansion and $\alpha$ is an adjustable parameter. By putting the variable change $y=(\alpha \gamma / 2)\left(\rho_{o} / \sigma_{o}\right)$, Eq. (1) can be written as

$$
\frac{1}{\rho_{o}^{2}+\left(\sigma_{o}^{2} / \alpha^{2}\right)}=\sqrt{\frac{2}{\pi}} \frac{\alpha}{2 \sigma_{o}^{2}} \sum_{n=0}^{N} a_{2 n}(\alpha) H_{2 n}\left(\frac{\rho_{o}}{\sigma_{o}}\right) e^{-\frac{\rho_{0}^{2}}{2 \sigma_{0}^{2}}} .
$$

So, in the input plane $\mathrm{z}=0$, the expression of the amplitude field in cylindrical coordinate system of Circular-Lorentz-Gaussian field which is introduced by Xu and G. Zhou [Y. Xu, and G. Zhou, 2019], is given by

$$
E_{n}^{\alpha}\left(\rho_{0}, z=0\right)=\frac{A_{0} \alpha}{\sqrt{2 \pi}} e^{-\left(\frac{1}{\left.\omega_{0}^{2}+\frac{1}{2 \sigma_{0}^{2}}\right)} \rho_{0}^{2}\right.} \sum_{n=0}^{N} a_{2 n}(\alpha) H_{2 n}\left(\frac{\rho_{o}}{\sigma_{o}}\right),
$$

where $\rho_{0}$ and $\mathrm{z}$ are the cylindrical coordinates, $\sigma_{0}$ is the width parameter of the Lorentz part, $\omega_{0}$ is the spot size of the fundamental Gaussian mode and $\mathrm{A}_{0}$ is the amplitude of the origin. To describe our proposing field, called Circular Lorentz-Bessel-Gaussian beams at $\mathrm{z}=0$, we will define it as follows

$$
E_{m ; n}^{\alpha}\left(\rho_{0}, z=0\right)=E_{n}^{\alpha}\left(\rho_{0}, z=0\right) J_{m}\left(\beta \rho_{0}\right),
$$

where $J_{m}$ is the $m$ th-order Bessel function of the first kind (here, considered as the beam-order) with $\beta=k \sin \phi \quad(\phi$ is a parameter of the transverse component of the wave vector) and $k=2 \pi / \lambda$ is the wave number associated with the wavelength $\lambda$. By the use of Eq. (3), the amplitude field of CLBGBs can be expended as 


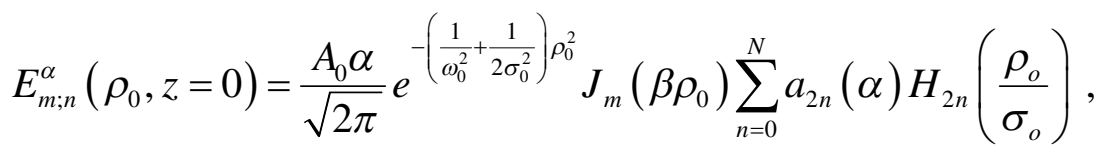

where the coefficients $a_{2 n}$ are functions of the parameter $\alpha$ and are given by (Schmidt, 1976)

$$
\begin{aligned}
a_{2 n}(\alpha) & =(-1)^{n} \frac{2}{2^{2 n} \sqrt{\pi}}\left[\frac{1}{n !} D_{-1}(1 / \alpha) e^{1 / 4 \alpha^{2}}\right. \\
& \left.+\sum_{s=1}^{n} \frac{2}{(2 s) !(n-s) ! \alpha^{2 s}}\left(D_{-1}(1 / \alpha) e^{1 / 4 \alpha^{2}}+\sum_{t=1}^{s}(-1)^{t}(2 t-3) ! ! \alpha^{2 t-1}\right)\right],
\end{aligned}
$$

with $D_{-1}$ is the parabolic cylindrical factor of order -1 , given in terms of the error function $\operatorname{erf}(\cdot)$ as follows

$$
D_{-1}(z)=\sqrt{\frac{\pi}{2}}[1-\operatorname{erf}(z / \sqrt{2})] e^{z^{2} / 4}
$$

Eq. (5) is a fundamental expression of the incident CLBGBs proposed in this study.

\section{Realization of CLG beams}

We propose here an experimental setup to realize our beams family. The detail of the realization of Hermite-Bessel-Gaussian beams is derived in Ref. (Soares et al. 2006). Note that these beams are non-diffracting waves, although the obtained CLBGBs are pseudo-non-diffracting ones. Basing of the work of Soares et al. (Soares et al. 2006), we consider a decomposition of Hermite-Bessel beams modulated by a Gaussian aperture. By using Eq. (4), one can realize the CLBGBs as a sum of Hermite-Bessel-Gaussian beams.

\section{Propagation of CLBGBs through an ABCD optical system}

The propagation of CLBGBs passing through a paraxial ABCD optical system is characterized by generalized Huygens-Fresnel diffraction of the form (Collins 1970)

$$
E_{m ; n}^{\alpha}(\rho, z)=\frac{A_{0}}{i \lambda B} e^{i k\left(z+\frac{D}{2 B} \rho^{2}\right)} \int_{0}^{\infty} \int_{0}^{2 \pi} E_{m ; n}^{\alpha}\left(\rho_{0}, z=0\right) e^{\frac{i k}{2 B}\left[A \rho_{0}^{2}-2 \rho \rho_{0} \cos \left(\theta-\theta_{0}\right)\right]} \rho_{0} d \rho_{0} d \theta_{0} .
$$

On substituting $E_{m ; n}^{\alpha}\left(\rho_{0}, 0\right)$ from Eq. (3) into Eq. (8) and applying the integral formula (Erdelyi 1954) 


$$
2 \pi J_{0}\left(\frac{k \rho \rho_{0}}{B}\right)=\int_{0}^{2 \pi} e^{-\frac{i k}{B} \rho \rho_{0} \cos \left(\theta-\theta_{0}\right)} d \theta_{0},
$$

with $J_{0}$ being the Bessel function of order zero, Eq. (8) becomes

$$
E_{m ; n}^{\alpha}(\rho, z)=\frac{A_{0} \alpha}{\omega_{0}^{2 m} \sqrt{2 \pi}} \frac{k}{i B} e^{i k\left(z+\frac{D}{2 B} \rho^{2}\right)} \sum_{n=0}^{N} a_{2 n}(\alpha) I_{n}
$$

where

$$
I_{n}=\int_{0}^{\infty} \rho_{0} e^{-(a+g) \rho_{0}^{2}} J_{m}\left(\beta \rho_{0}\right) J_{0}\left(b \rho_{0}\right) H_{2 n}\left(\sqrt{2 g} \rho_{0}\right) d \rho_{0},
$$

with $b=\frac{k \rho}{B}, g=\frac{1}{2 \sigma_{0}^{2}}$ and $a=\frac{1}{\omega_{0}^{2}}-\frac{i k A}{2 B}$.

Earlier, Belafhal et al., 2020 have investigated the following result

$$
\begin{aligned}
\int_{0}^{\infty} t^{2 q+\frac{1}{2}} e^{-(\alpha+\eta) t^{2}} J_{\chi}(\lambda t) J_{\mu}(\delta t) H_{2 p}(\sqrt{2 \eta} t) d t & =\frac{(2 p) ! A_{\mu,-\frac{1}{4}}\left(\frac{\lambda}{2}\right)^{\chi} \Gamma\left(\alpha_{q}^{-\frac{1}{4}}\right)}{(-1)^{p}(2 \eta)^{\frac{1}{4}}(\alpha+\eta)^{\alpha_{q}^{-\frac{1}{4}}} \chi ! p !} \\
& \times F_{A}^{(3)}\left(\alpha_{q}^{-1 / 4},-p,-,-; \chi+1, \mu+1,1 / 2 ; \kappa, \zeta, \xi\right),
\end{aligned}
$$

with $\left[R(\mu)>-1, R\left(q+\frac{2 \mu-1}{4}\right)>-1, R(\alpha+\eta)>2 \eta\right]$,

where

$$
\kappa=\frac{2 \eta}{\alpha+\eta}, \zeta=-\frac{\lambda^{2}}{4(\alpha+\eta)}, \xi=-\frac{\delta^{2}}{4(\alpha+\eta)}, \alpha_{q}^{v}=q+\frac{\chi+\mu}{2}+v+1, A_{\mu, v}=\frac{\beta^{\mu} \eta^{\mu+\frac{1}{2}}}{2^{\mu-v+\frac{1}{2}} \mu !}
$$

and $F_{A}^{(3)}(x, y, z)$ is a summation formula of a general triple hypergeometric series, or so-called "Lauricella's triple hypergeometric function". By setting the above integral parameters as $v=-\frac{1}{4}, q=\frac{1}{4}, \chi=m$ and $\mu=0$, and applying the last integral representation, Eq. (10) becomes 


$$
\begin{aligned}
E_{m ; n}^{\alpha}(\rho, z) & =\frac{A_{0} \alpha k}{2 i \sqrt{2 \pi} B} \frac{(\beta / 2)^{m} \Gamma\left(\frac{m}{2}+1\right)}{(a+g)^{\left(\frac{m}{2}+1\right)} m !} e^{i k\left(z+\frac{D}{2 B} \rho^{2}\right)} \sum_{n=0}^{N} a_{2 n}(\alpha) \frac{(2 n) !}{(-1)^{n} n !}, \\
& \times F_{A}^{(3)}\left(\frac{m}{2}+1,-n,-,-; 1, m+1,1 / 2 ; u, v, w\right),
\end{aligned}
$$

where $u=\frac{2 g}{a+g}, v=-\frac{\beta^{2}}{4(a+g)}$, and $w=-\frac{b^{2}}{4(a+g)}$.

Eq. (13) is the main result of this paper that gives the final analytical expression for the paraxial transverse of the Circular Lorentz-Bessel-Gaussian beams propagating through an ABCD optical system.

\section{Numerical examples}

In this Section, by using our analytical result given by Eq.(13), the propagation properties of CLBGBs in free space was investigated by some numerical examples. We will examine in the following the contour graphs and the coressponding curves of the normalized intensity distribution of the zero and first-orders CLBGBs propagating through free space versus the $\mathrm{x}$-axis and with different parametrers such as the Lorentzian and Gaussian waists, the beam-order $m$ and the parameter $\beta$. The calculation parameters for each figure are chosen to be: $\lambda=0.8 \mu \mathrm{m}$ and the matrix elements are: $\mathrm{A}=1, \mathrm{~B}=\mathrm{z}, \mathrm{C}=0$ and $\mathrm{D}=1$.

Fig. 1 represents the graphical results to display the normalized intensity distribution of CLBGBs at a received plane $\mathrm{z}$ and for different Lorentzian waists $\sigma_{0}$. The other parameters are: $z=2 z_{R}$ ( $z_{R}=\pi \omega_{0}^{2} / \lambda$ denotes the Rayleigh length of the Gaussian part), $\omega_{0}=2 \mathrm{~mm}$, and $\beta=2$. It can be deduced that, in the small Lorentzian waist values $\left(\sigma_{0} \leq 0.3 \mathrm{~mm}\right)$, the normalized intensity profile of CLBGBs is close to a flattop Gaussian profile (see Fig. 1a); however, as $\sigma_{0}>0.3$, the central dark or bright part gradually appears and it can be controlled by varying the value of $\sigma_{0}$ (see Fig. 1 (b-d)). It can also obseved that the intensity peaks of the dark or bright spots increases with $\sigma_{0}$. This result demonstrated that, the Lorentzian waist has a clear influence on the normalized intensity distribution of CLBGBs. 

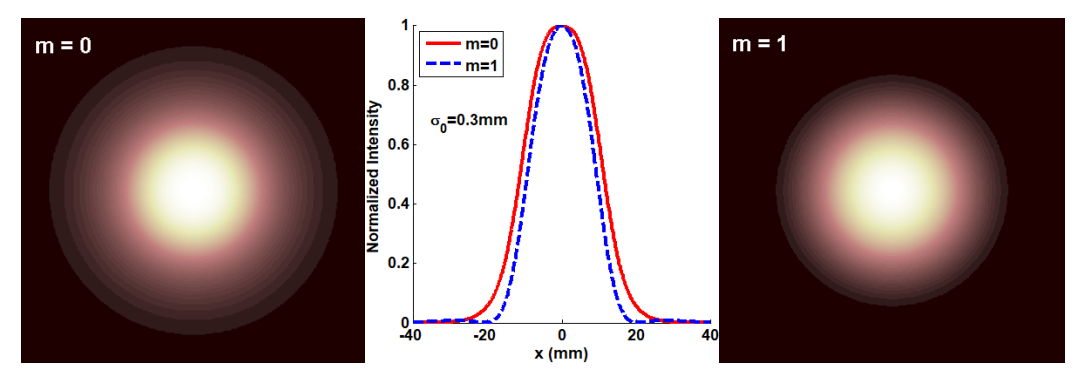

(a)
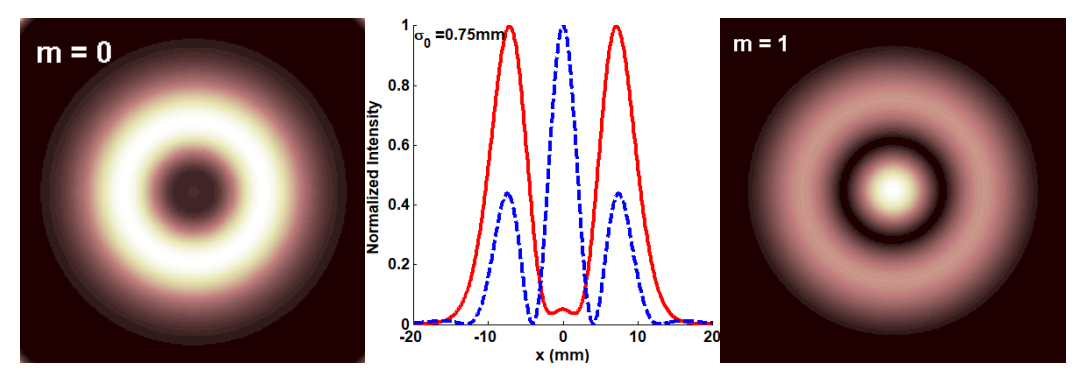

(b)
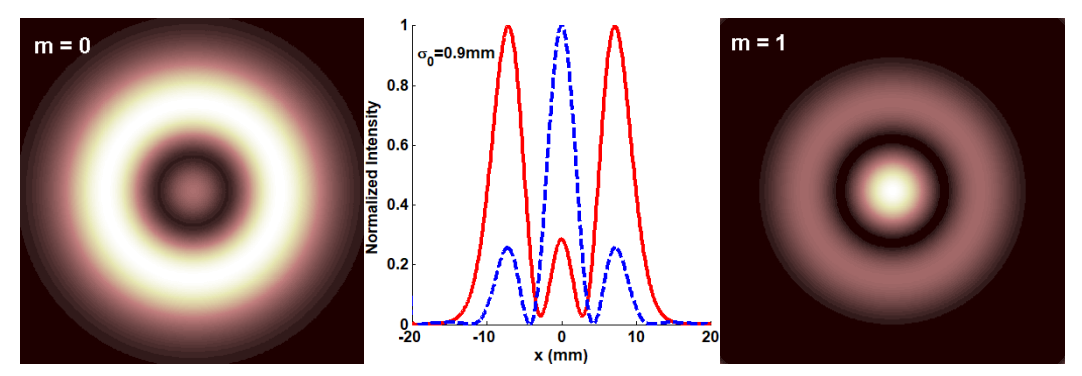

(c)
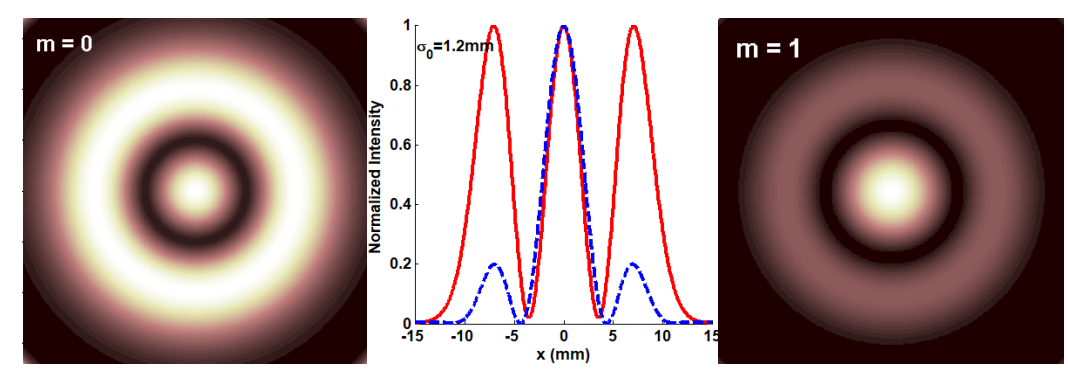

(d)

Figure 1: Contour graphs and coressponding curves of the normalized intensity distribution of a $m$ th-order CLBGB at $\mathrm{z}=2 \mathrm{Z}_{\mathrm{R}}$, with $\beta=2, \omega_{0}=2 \mathrm{~mm}$ and for the different values of $\sigma_{0}$ :

(a) $\sigma_{0}=0.3 \mathrm{~mm}$, (b) $\sigma_{0}=0.75 \mathrm{~mm}$, (c) $\sigma_{0}=0.9 \mathrm{~mm}$, and (d) $\sigma_{0}=1.2 \mathrm{~mm}$.

Similarly, Fig. 2 illustrates the normalized intensity distributions of CLBGBs for different Gaussian waists and with a fixed Lorentzian waist $\left(\sigma_{0}=2 \mathrm{~mm}\right)$, with the other parameters are similar to that of Fig. 1. The beam profiles of Fig. 2 are close to those of Fig. 1 exept that the intesity profiles in Fig. 2 become more concentrating arround the central beam. 
Also, it can be seen that, if $\omega_{0}<0.3 \mathrm{~mm}$, the intensity distribution profile of CLBGBs is like to the well-known Gaussian profile as illustrated in Fig. 2a. Then, if $\omega_{0} \geq 0.75 \mathrm{~mm}$, the intensity profile will oscillate to give dark or bright spots with increasing $\omega_{0}$.
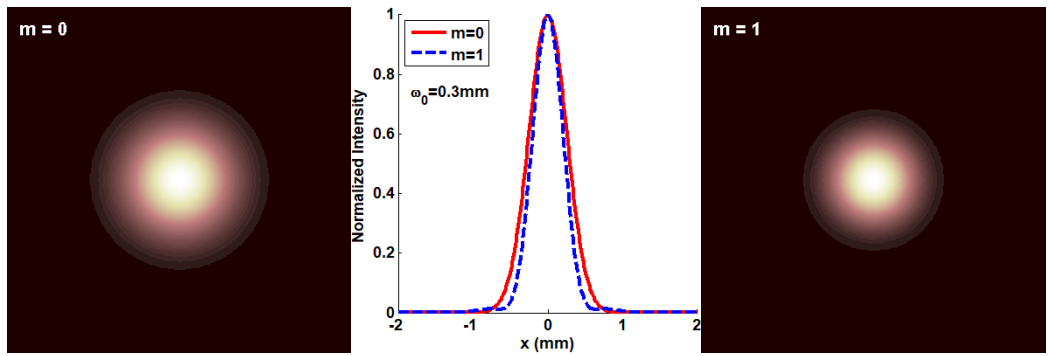

(a)
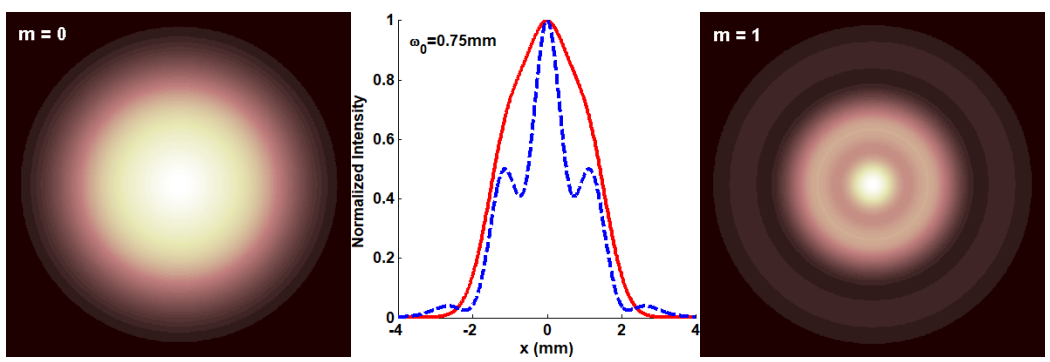

(b)
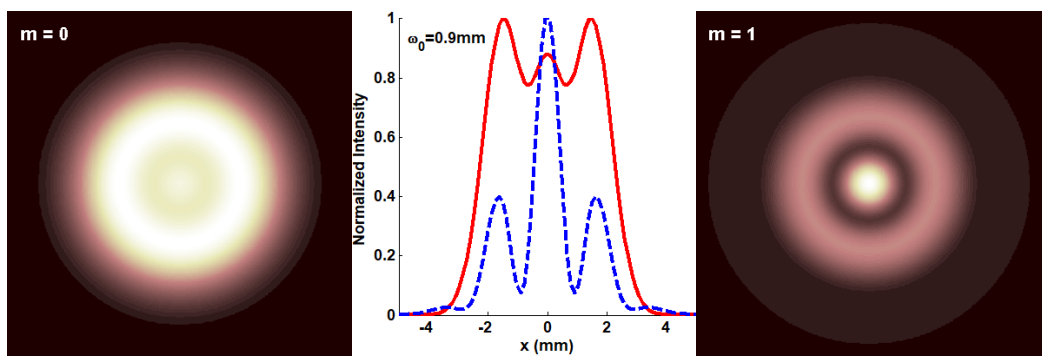

(c)
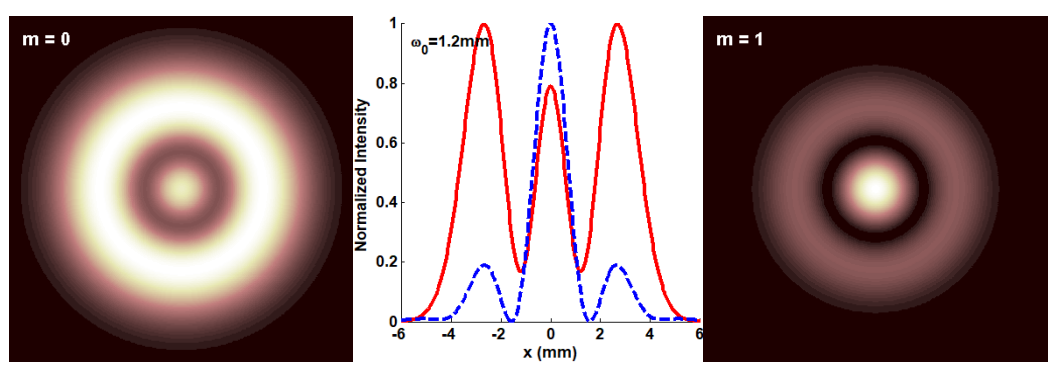

(d)

Figure 2: Contour graphs and the coressponding curves of normalized intensity distribution of a $m$ th-order CLBGB at $\mathrm{z}=2 \mathrm{z}_{\mathrm{R}}, \beta=2, \sigma_{0}=2 \mathrm{~mm}$ and for the different values of $\omega_{0}$ :

(a) $\omega_{0}=0.3 \mathrm{~mm}$, (b) $\omega_{0}=0.75 \mathrm{~mm}$, (c) $\omega_{0}=0.9 \mathrm{~mm}$, and (d) $\omega_{0}=1.2 \mathrm{~mm}$.

The influence of $\beta$-parameter on the normalized intensity distribution of CLBG beams in the reference plane $\mathrm{z}=2 \mathrm{z}_{\mathrm{R}}$, is demonstrated in Fig. 3. The calculation parameters are chosen as Fig. 1. 

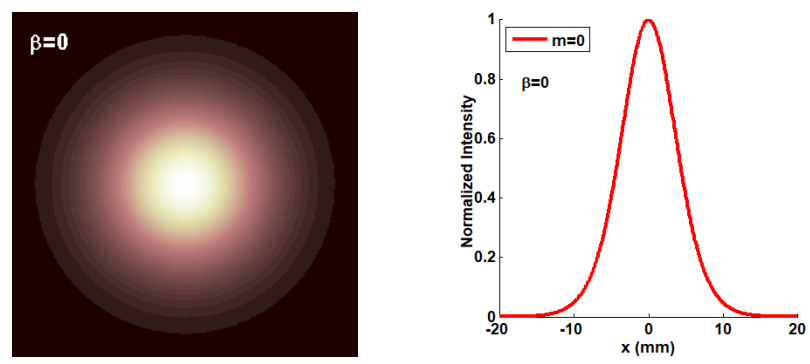

(a)
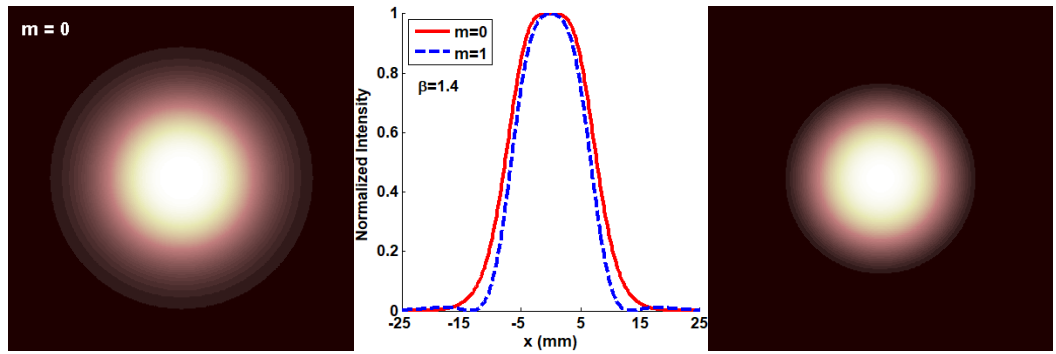

(b)
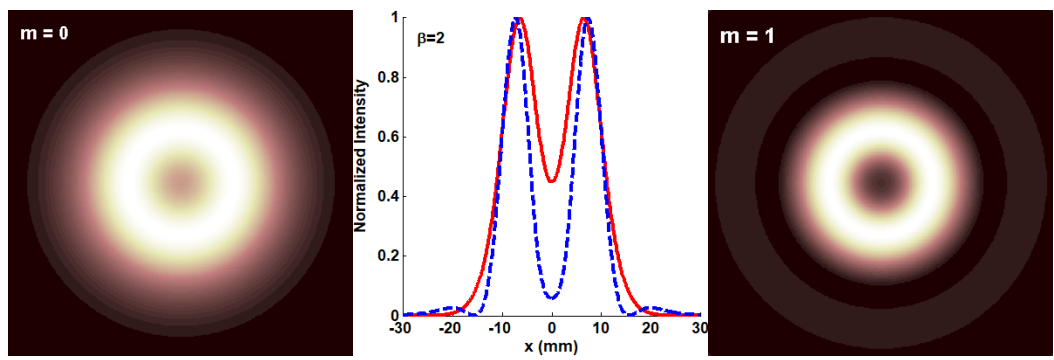

(c)
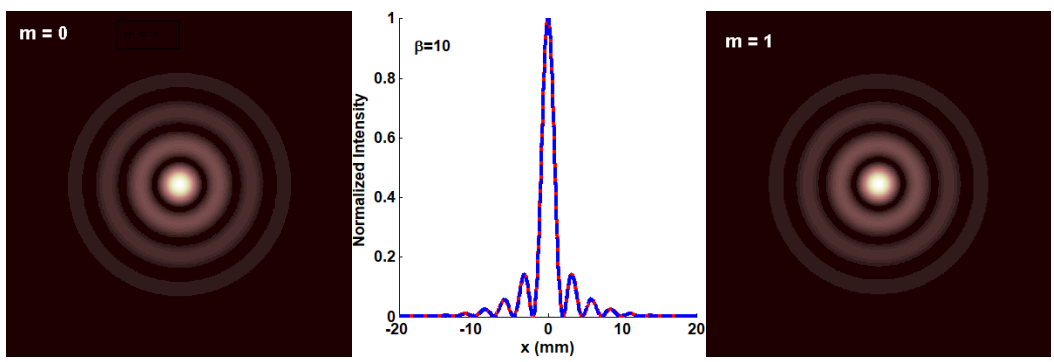

(d)

Figure 3: Contour graphs and the coressponding curves of the normalized intensity distribution of a $m$ th-order CLBGB with $\mathrm{z}=2 \mathrm{z}_{\mathrm{R}}, \omega_{0}=2 \mathrm{~mm}$, and $\sigma_{0}=0.5 \mathrm{~mm}$ and for different values of $\beta$ :

(a) $\beta=0$, (b) $\beta=1.4$, (c) $\beta=2$, and (d) $\beta=10$.

From the plots of Fig. 3, we can conclude that the normalized inrensity distribution of CLBG beams given as $\beta=0$ is similar to Fig. 1a of Ref. (Xu and Zhou, 2019), which confirms that it is a special case of our work. When the parameter $\beta$ varies from 0 up to 2 , the corresponding contour/curves are similar to those given in the previous Figs. 1 and 2, however at $\beta>1$, an oscillating behaviour appears and becomes more and more evident at large values of $\beta$ (see Fig. 
3d). This last property makes us believe that, the CLBG beams are possible to obtain a stable propagation state, when $\beta$ is large enough.

Fig. 4 shows the normalized intesity distribution for bothe the zero and the first orders of Circular Lorentz-Bessel-Gaussian beams at different propagation distances $\mathrm{z}$ and with $\beta=2, \omega_{0}=2 \mathrm{~mm}$ and $\sigma_{0}=0.5 \mathrm{~mm}$.
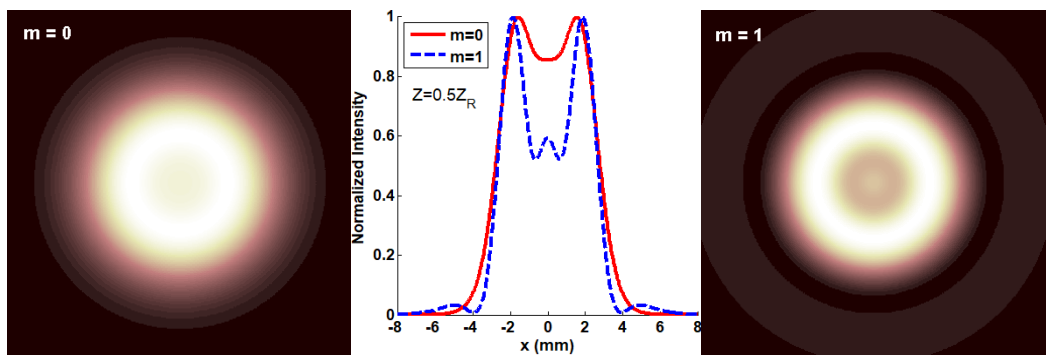

(a)
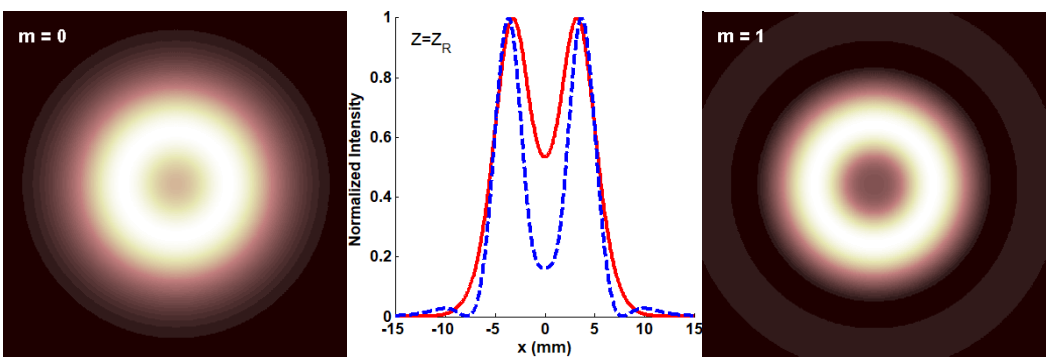

(b)
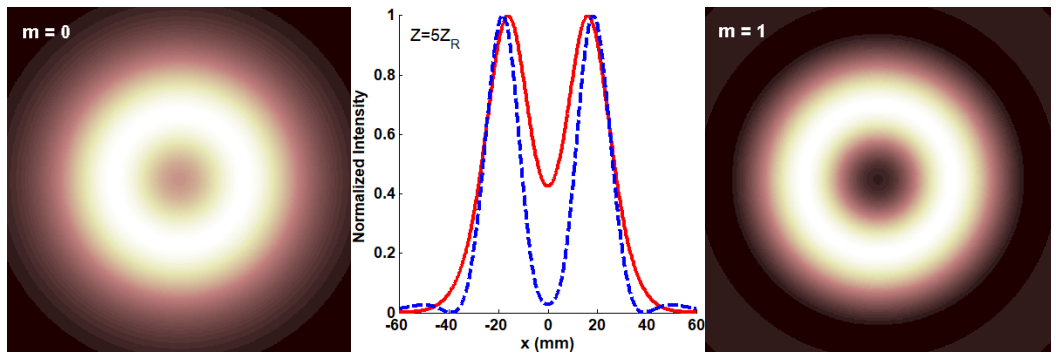

(c)
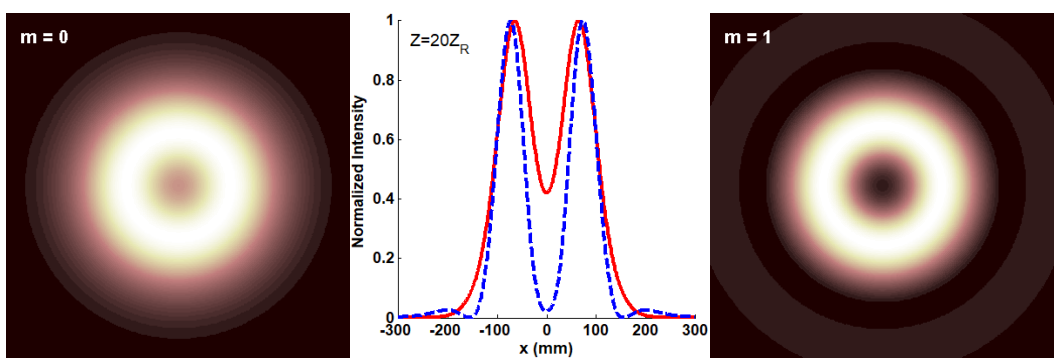

(d)

Figure 4: Contour graphs and the coressponding curves of the normalized intensity distribution of a $m$ th-order CLBGB, with $\omega_{0}=2 \mathrm{~mm}, \sigma_{0}=0.5 \mathrm{~mm}$ and $\beta=2$ and at several selected distances $\mathrm{z}$ :

(a) $\mathrm{z}=0.5 \mathrm{z}_{\mathrm{R}}$, (b) $\mathrm{z}=\mathrm{z}_{\mathrm{R}}$, (c) $\mathrm{z}=5 \mathrm{z}_{\mathrm{R}}$, and (d) $\mathrm{z}=20 \mathrm{z}$. 


\section{Conclusion}

In summary, the normalized intensity distribution of CLBGBs through an ABCD paraxial optical system has been investigated by applying the generalized Huygens-Fresnel diffraction integral of the form Collins formula and the expansion of the Lorentz distribution in terms of the complete orthonormal basis set of the Hermite-Gauss modes. The closed-form formula of the output complex amplitude distributions of the light field has been derived as a new complex function that so-called "Lauricella's triple hypergeometric function". By using our analytical formulae, numerical calculations have been performed to illustrate the propagation of CLBGBs in free space optical system. Our results show that the normalized intensity of the considered beams is more sensitive to the changes in the mentioned parameters, in particular the parameter of Bessel part $\beta$. 


\section{References}

Gawhary O. E., Severini S.: Lorentz beams and symmetry properties in paraxial optics. J. Opt. A 8, 409-414 (2006).

Gawhary O. E., Severini S.: Lorentz beams as a basis for a new class of rectangular symmetric optical fields. Opt. Commun. 269 (2), 274-284 (2007).

Dumke W. P., The angular beam divergence in double-heterojunction lasers with very thin active regions. IEEE J. Quantum Electron. 11 (7), 400-402 (1975).

Naqwi A., Durst F.: Focus of diode laser beams: a simple mathematical model. Appl. Opt. 29 (12), 1780-1785 (1990).

Yang J., Chen T., Ding G., Yuan X.: Focusing of diode laser beams: a partially coherent Lorentz model. Proc. SPIE 6824, 68240A (1-8) (2008).

Xu Y., Zhou G.: Circular Lorentz-Gauss beams. J. Opt. Soc. Am. A 36, 179-185 (2019).

Zhou G., Propagation of a partially coherent Lorentz-Gauss beam through a paraxial ABCD optical. Optics Express 18(5), 4637-4643 (2010a).

Zhou G, Propagation of a Lorentz-Gauss beam through a misaligned optical system. Optics Commun. 283, 1236-1243 (2010b).

Zhou G., Fractional Fourier transform of Lorentz-Gauss beams. J. Opt. Soc. Am. A 26, 350-355 (2009a).

Du W., Chengliang Zhao, Cai Y.: Propagation of Lorentz and Lorentz-Gauss beams through an apertured fractional Fourier transform optical system. Optics and Lasers in Engineering 49, 25-31 (2011).

Muhsin C.G., Eyyuboğlu H.T.: Irradiance fluctuations of partially coherent super Lorentz Gaussian beams. Optics Commun. 284, 4857-4861 (2011).

Zhao C., Cai Y.: Paraxial propagation of Lorentz and Lorentz-Gauss beams in uniaxial crystals orthogonal to the optical axis. J. Mod. Opt. 57 (5), 375-384 (2010).

Zhou G., Beam propagation factors of a Lorentz-Gauss beam. Appl. Phys. B 96, 149-153 (2009b).

Zhou G., The beam propagation factors and the kurtosis parameters of a Lorentz beam. Optics \& Laser Technology 41, 953-955 (2009c).

Rui F., Zhang D., Ting M., Gao X., Zhuang S.: Focusing of linearly polarized Lorentz-Gauss beam with one optical vortex. Optik 124, 2969-2973 (2013). 
Schimpf D. N., Schulte J., Putnam W. P., Kartner F. X.: Generalizing higher-order Bessel-Gauss beams: analytical description and demonstration. Optics Express 20(24), 26852- 26867 (2012).

Fahrbach F.O., Simon P., Rohrbach A.: Microscopy with self-reconstructing beams. Nat. Photonics 4, 780-785 (2010).

Planchon T. A, Gao L., D. Milkie E, Davidson M. W, Galbraith J. A, Galbraith C. G, Betzig E.: Rapid three-dimensional isotropic imaging of living cells using Bessel beam plane illumination. Nat. Methods 8, 417-423 (2011).

Duocastella M., C. B. Arnold C.B.: Bessel and annular beams for materials processing. Laser Photon. Rev. 6, 607-621 (2012).

Garcés-Chávez V., McGloin D., Melville H., Sibbett W., Dholakia K.: Simultaneous micromanipulation in multiple planes using a self-reconstructing light beam. Nature 419, 145-147 (2002).

Li D., Imasaki K.: Vacuum laser-driven acceleration by two slits-truncated Bessel beams. Appl. Phys. Lett. 87, 0911061-1-3 (2005).

Belafhal A., El Halba E. M., Usman T.: An Integral Transform Involving the Product of Bessel Functions and Whittaker Function and Its Application. Mathematics Subject Classification, Int. J. Appl. Comput. Math. 6, 177-188 (2020).

Schmidt P.P.: A method for the convolution of line shapes which involve the Lorentz distribution. J. Phys. 9, 2331-2339 (1976).

Collins, S.A.: Lens-system diffraction integral written in terms of matrix optics. J. Opt. Soc. Am. 60, 1168-1177 (1970).

Soares W. C., Caetano D. P., Hickmann J. M.: Hermite-Bessel beams and the geometrical representation of nondiffracting beams with orbital angular momentum. Optics Express 14 (11), 4577-4582 (2006).

Erdelyi A., W. Magnus, F. Oberhettinger: Tables of Integral Transforms (McGraw-Hill, 1954). 